\title{
MODULARIZATION BUSINESS CASE: PROCESS FLOWCHART AND MAJOR CONSIDERATIONS
}

\author{
William J. O’Brien ${ }^{1}$; James T. O’CONNOR ${ }^{2}$; Jin Ouk $\mathrm{CHOI}^{3 *}$ \\ $1 \& 2$ The Univ. of Texas at Austin, Dept. of Civil, Architectural and Environmental Engineering, 1 \\ Univ. Station C1752, Austin, TX 78712 \\ ${ }^{3}$ Iowa State University, Dept. of Civil, Construction and Environmental Engineering, 394 Town \\ Engineering, Ames, IA 50011 \\ *Corresponding author's e-mail: choi.jinouk@gmail.com
}

\begin{abstract}
Modularization is a well-known method of enhancing project value by exporting a portion of site work to one or more local or distant fabrication or assembly shops/yards. Still, the industry is in need of additional guidance on how to more effectively exploit modularization. To help achieve wider and more effective use of modularization, the researchers and the Construction Industry Institute's (CII) Research Team 283 develops here a new modularization business case process for developing the modularization drivers (and for determining the degree to which modularization will be implemented). The result is an optimal decision-making process as these drivers are compared with the owner's objectives and evaluation criteria for cost, schedule, risk and other project objectives. This paper presents this new modularization business case process and lays out the major considerations that pertain to per-project phases. The findings provide guidelines along with a flowchart that should impose rigor on the decision process, helping owners and avoiding poor outcomes.
\end{abstract}

\section{KEYWORDS}

Modularization; Modular and offsite construction; Modularization business case analysis; Optimal decision-making process

\section{INTRODUCTION}

Modularization is a method of enhancing project value by exporting a portion of site work to one or more local or distant fabrication or assembly shops/yards. Though it has been utilized by the industry for centuries, the industry is presently having difficulty achieving desired levels of modularization or seeing the maximum benefit that modularization could provide. To help achieve wider and more effective use of modularization, the industry needs additional guidance on how to more effectively exploit modularization. There are several reports that attempt to assess modularization business case analysis through case studies (Jameson 2007; Wu and Lu 2013). However, most of the publically available reports are limited to case studies or lessons learned and are limited to optimizing local problems. To solve this problem and to help achieve wider and more effective use of modularization, the researchers and the Construction Industry Institute's (CII) Research Team 283 set out to develop a new modularization business case 
process for developing modularization drivers (and for determining the degree to which modularization will be implemented). The aim was to come up with an optimal decision-making process, as these drivers can be compared with the owner's objectives and evaluation criteria for cost, schedule, risk and other project objectives. To achieve this goal, the researchers posed two research questions.

- How can the benefits and costs of the modular approach be assessed?

- How does the Business Case fit within the context of project development?

The CII publications that provide relevant information on Modularization Business Case Analysis are:

1) Research Summary 283-1, "Industrial Modularization: How to Optimize? How to Maximize?" (CII 2012)

2) Implementation Resource 283-2, "Industrial Modularization: Five Solution Elements" (CII 2013)

3) Research Report 283-11, "Industrial Modularization: How to Optimize? How to Maximize?" (O'Connor et al. 2013)

The scope of the research concerns primarily the industrial sub-sector, including among others process and manufacturing facilities such as offshore facilities, petro-chemical plants, power plants, and pharmaceutical plants.

\section{RESEARCH METHODS}

\section{Business Case Process Methodology}

This study was carried out in four steps: 1) review and discuss different corporate approaches, 2) draft a multi-phase process, 3) refine with respect to phase context and data needed, 4) finalize recommended process and 5) validation by external experts.

1. Review and Discuss Different Corporate Approaches. In order to create a business case process that supports broader modularization, the research team discussed existing business case processes from different companies. The goal was to select and borrow the most suitable elements of each process in order to incorporate them in a new business case process. The new process focus on the early phases of a project where a big impact can be made when modularization is taken into account. Currently, most of the work in the early phases of a project is done internally by the owner. Therefore there is a risk that, without explicitly knowing about modular options, modularization possibilities could be excluded.

2. Draft a Multi-Phase Process. Because the business case process is inherently a decision driven process, a decision tree procedure was created. The multi-phase procedure includes elements such as high-level technical considerations, site characteristics, weather, schedule, fabrication, modularization drivers, module geometries, execution, and costs. The goal of increased use of modularization is more likely to be reached if all steps are carefully studied and acted upon by the appropriate organizations.

3. Refine with Respect to Phase Context and Data Needed. Because different decisions have to be made at different points of the project, the decision tree procedure has to be developed and followed in different ways. The research team identified which decisions are essential at the Opportunity Framing Phase, at the Assessment Phase, and at the Selection Phase. The differentiation is made so that organizations (Owner and/or Contractor teams) are aware that different efforts are necessary for each business case phase.

4. Finalize Recommended Process. Further discussion and analysis of the proposed process in terms of scope, accuracy, and applicability of the different elements at different stages is 
performed in order to create a concise, efficient tool. It is critical to have such a sound and integrated process so that the stakeholders who have the power to decide what the level of modularization in a project will be can make that decision with enough confidence in the accuracy of the process and the elements upon which it is based.

5. Validation by External Experts. 14 external experts from various companies were invited for the review. The validation committee had about a month to review the draft of research findings and returned their individual feedback. The researchers and the research team members went through every comment received item-by-item from the validation committee and reacted to them according to three response types: 1. responded, 2. disagreed w/neglected, or 3. already in place/no change needed. Detail of responses to the comments can be found in CII Research Report 283 (O'Connor et al. 2013).

\section{Research Team \& External Validation Committee Background}

The modularization business case process is part of the research results produced by CII RT283. The 21-member team was made up of experts from the construction industry and academic institutions. RT 283 has a total of 450+ years of experience and has been involved in 170+ modular projects constructed in the last 5 years in 13 countries. The RT had two members from academia; industry representatives ranged from five owners, eight contractors and design firms, and six fabricators. The external validation committee, consisting of 14 volunteers from the construction industry, validated the research product drafts on modularization business case process. A survey found that the committee has 458+ years of experience and, in the last 5 years, have undertaken work on $123+$ modular projects.

\section{RESULTS - BUSINESS CASE FLOWCHART AND MAJOR CONSIDERATIONS PER PHASE}

The modular execution business case analysis provides a rigorous method to be applied in answering the following fundamental project execution questions:

1. Should this project adopt a modular execution?

2. If so, what is the optimum proportion of work hours that should be moved offsite in the module scope?

In addition, the business case analysis provides a process for developing the modularization drivers (and for determining the degree to which modularization will be implemented). The result is an optimal decision-making process as these drivers are compared with the owner's objectives and evaluation criteria for cost, schedule, risk and other project objectives. This will also enable, as the project progresses, the module scope to be refined, formalizing the process of identifying optimum and maximum module sizes and weights.

The business case for or against modularization is typically undertaken in successive stages with several options being evaluated. As the project develops and as more details become available, the business case decision process will develop from an initial analysis utilizing high-level information to more detailed analyses using more and more project-specific data until the execution model has been refined to a detailed execution plan.

This paper details an ideal model for business case analysis to develop the optimum degree of modularization appropriate for a project. If analysis is undertaken later in the project than prescribed in this paper, the steps outlined can be undertaken in an accelerated fashion, though the opportunity for optimum use of modularization may have passed. This paper is presented in two parts. First, a model business case decision flow chart is presented, followed by a detailed 
discussion of analysis activities related to modularization by phase. This detailed analysis makes specific reference to the decision flow chart.

\section{Model Decision Flowchart}

Consideration of modularization options should be guided by past experience as well as creative identification of options and new approaches given changes in technology, project specific demands, etc. When specific modularization approaches have been identified, they can be analyzed for benefits to make a go/no go decision for the modular approach. This assessment to embrace modularization can be further refined to make specific decisions regarding the degree of modularization to adopt on the project, optimum module sizes, etc. Evaluation of a specific modular approach can be undertaken using the multi-step flowchart that details the major considerations and decision points as shown in Figure 1. The flowchart detailed in Figure 1 is meant to be repeatedly utilized during each of the major project decision phases, typically in parallel with the development of stick build and modular option estimate data, adding detail in each phase. In general, a go/no go decision would be made during the Assessment Phase or possibly the Selection Phase depending on the particular characteristics of the project. It may also be possible to make a late decision in the Basic Design Phase though, for most projects, it is unlikely that optimum project benefits will be obtainable at this stage. However, new information may be developed that could change the case for or against the modular approach so it remains important to address the question "is modularization viable" (step 11 in the Figure 1) in each phase.

To develop the business case (as well as to generate specifications for execution to achieve the planned benefits), it is important to evaluate the costs and benefits of moving work hours from the on-site work scope into module fabrication yards as modules (including schedule implications) (steps 2-4). Analysis of these costs and benefits will formulate drivers for and against modularization (step 5). By comparing the benefits and costs identified in step 5 with the project's objectives and evaluation criteria, detail specifics of the module scope (including numbers and size) can be developed optimizing the module scope. As a part of the information developed on site specific factors and module yards, the project execution strategy will be developed (steps 6-9). These in turn support development of a cost estimate (step 10) that allows the viability decision to be made/confirmed (step 11). The cost estimate should allow comparison of modular versus stick built approaches (typically with a differential cost analysis) following that outlined above.

\section{Major Steps and Considerations by Project Phase}

Effective modularization requires analysis in all phases of a project, from the initial conception through detailed design and into procurement and construction. Summarized below are key actions to be considered for each phase of a project. Figure 2 details typical project phases as delineated by the research team and their correspondence to typical terminology used by different subsectors in the industry, whether decision making in a gated process (G1-G5), project estimate level, of FEED (Front End Engineering Design) /FEL (Front End Loading).

Opportunity Framing

- Main Activity: Strategic business assessment - determining drivers, cost and revenue potential. 
- Phase Goals: Concentrate on strategic fit of the project into the company's business portfolio with a high level analysis of the Project potential. In particular: technology / market potential / plant location prospects / preliminary timing to market / etc.

- Modularization Goal If requested, identify the potential execution risks for constructing the project in the location(s) under consideration and determine whether there is the potential for significant benefits through adopting a modular approach that may impact the project's go / no go decision.

- Methodology Keys to Success: Keep options open until proven to provide no benefit to the project.

- Pitfalls: Avoid too much detail or assumptions that cannot be substantiated.

Assessment

- Main activity: Identify major technical details of project, list of alternatives, constraints and key investigations to consider.

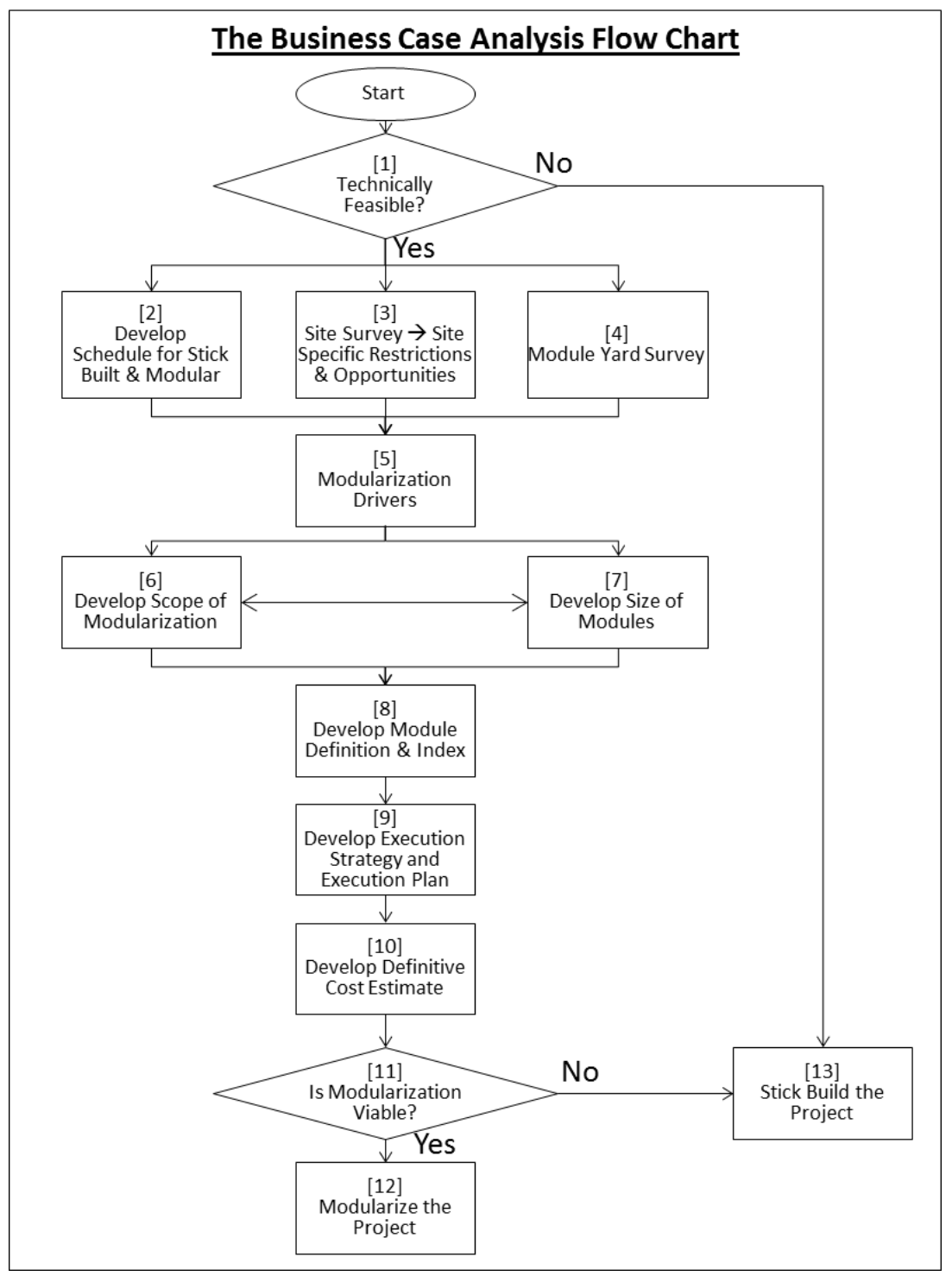

Figure 1. Business Case Analysis Flow Chart 


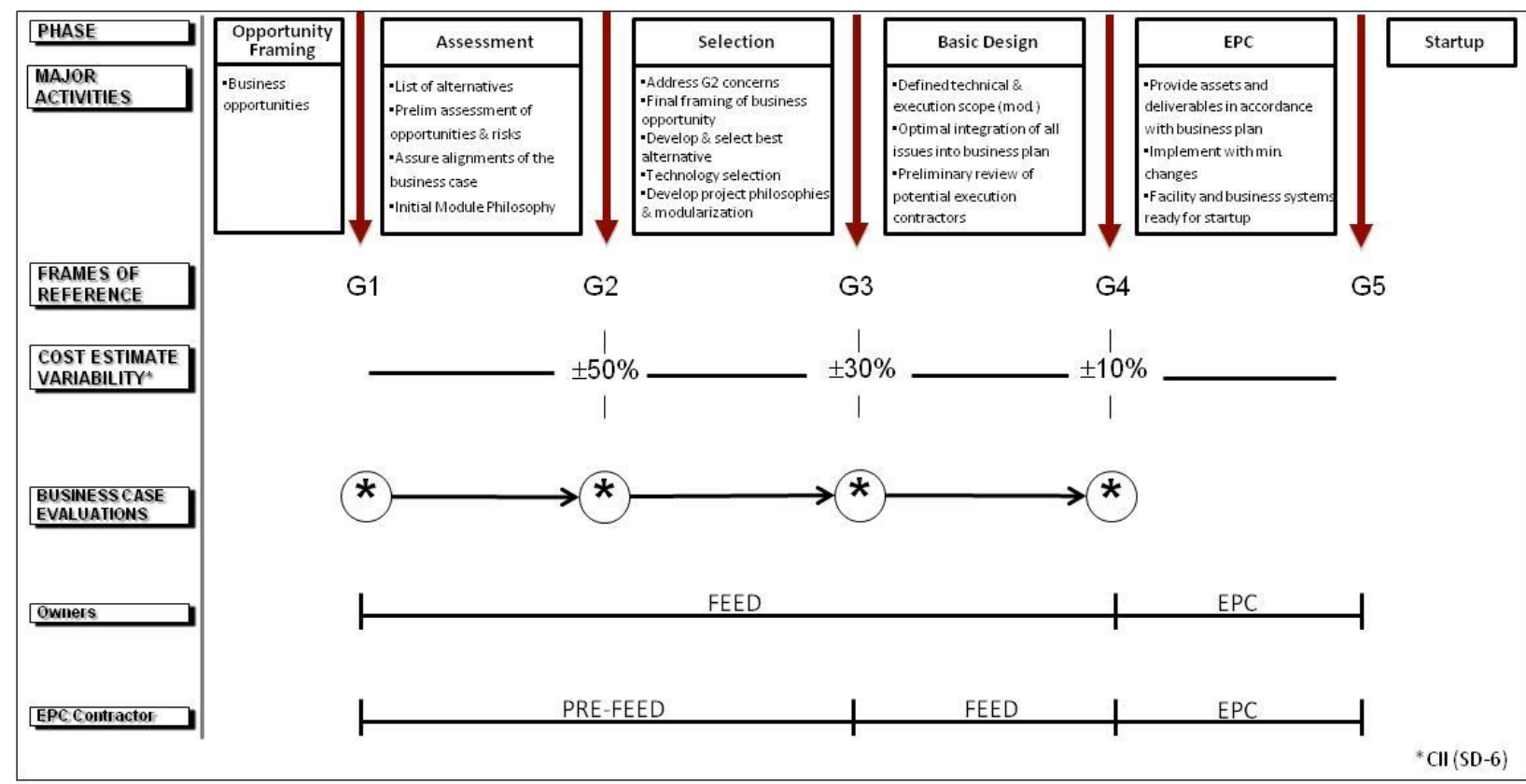

Figure 2. Typical Project Phases

- Phase Goals: (1) Address previous phases' concerns. (2) Final framing of the business opportunity. (3) Identify process alternative (4) Identify options from a project perspective. (4) Discuss technology and project philosophies (including modularization).

- Modularization Goal: Identify the modularization concept as a viable option to the standard Stick build construction for achieving project goals such as reducing overall cost and increasing N PV (Net Present Value). Show potential benefit of modularization and obtain approval to continue study of module options in next phase, even if it is only pre-assembly of parts of the facility.

- Methodology: Develop a high level risk /return type analysis based on key module drivers identified in the Project risk analysis. Optimal extent of modularization would be based on the strength of the drivers compared with the project's objectives and are obtained by a high level assessment of modularization potential in terms of high / medium / low percentages of work hours moved offsite. Use contractor or specialist engineering to supplement module experience if required. Develop set of module questions as basis for further analysis and develop critical milestones for decision making to capture maximum potential benefit. Begin systematic approach to module analysis using the flow chart if no other internal analysis system exists at this stage (if internal systems exist, they should be audited against the recommended steps in this paper). (Figure 1)

- Keys to Success: Tie module potential to NPV, improved ROI (return on investment) or other critical success metric. Consider progressing, the project as a modular project, because as it is relatively easy to convert a modular project to stick built, but not the reverse.

- Pitfalls: Owner / Contractor unfamiliarity with module concepts, as well as pre-conceived notions and prejudices potentially reducing benefit options too early. Not identifying potential additional budget needs for earlier engineering design efforts and 3rd party vendor information. 


\section{Selection}

- Main Activity: Further definition and selection of process and equipment alternatives, cost and schedule to support discussions on Project approval and funding.

- Phase Goals: (1) More accurate cost /schedule. (2) Set up of Project controls. (3) Better definition of systems - begin shift from trending to specific vendor information.

- Modularization Goal: Continue development of site layout and modules based on a clean page re-analysis rather than use of an historic stick build configuration where such changes look promising. Begin review of module fabrication yard sites and erection options.

- Keys to success: Owner has identified and fostered a Module Team to continue the analysis. Owner has supplemented his module experience level with additional technical support where required. Module options are identified that when compared will provide idea of best option. Plant layout and equipment configurations remain fluid for module option analysis. Additional module characteristics / details surface new questions. Old module questions get revisited with more detail and some are closed out. Early identification of 'early works' that may need to be designed and constructed in the Basic Design or early in the Execution Phases (such as construction jetties for receiving modules and heavy equipment).

- Pitfalls: Using stick build layout to define modules thereby reducing benefit potential of modularization (often significantly). Starting module analysis in this Phase without first reviewing necessary steps identified in earlier phases. Inability to accept conclusion that modularization may require additional upfront technical support for a longer duration. Failure to involve piping and structural design groups.

Basic Design

- Main Activity: Define optimal technical and execution scope. Integration of all issues into the final business plan (including modularization).

- Phase Goals: (1) Best module case vetted through all disciplines and project groups and incorporated into logic driven schedule, Project Execution Plan and Construction Plan for EPC (Engineering, Procurement and Construction) contractor follow through. (2) Process design and supporting equipment and systems developed. (3) Long Lead equipment identified with efforts initiated to procure in a timely manner. Develop cost benefit analyses to firm up decision making, and develop project estimate and control budgets.

- Modularization Goal: Further develop optimal module case identified in previous Phase analyses and solidify by the middle of this phase.

- Keys to Success: Module team actively interacting with Structural, Piping and Mechanical disciplines as well as Procurement and Construction to ensure module fab yard needs can be met. Creative approach to obtaining critical technical information where needed to keep modules design off critical path.

- Pitfalls: Failure to correctly evaluate module fab yard needs and material timing requirements and make adjustments on the front end of the engineering deliverables and procurement schedules.

\section{Post G4 Phase: EPC (Engineering, Procurement and Construction)}

Subsequent to development of the business case from Assessment through Basic Design, principal activities for the EPC phase are to ensure execution following the recommendations previously developed.

- Main Activity: Deliver Project per business plan 
- Phase Goals: (1) Provide assets (design and construct) in accordance with final business plan. (2) Implement with minimum changes. (3) Provide facility ready for start-up at the end of this phase.

- Modularization Goals: Execute module plan as developed in Basic Design.

- Keys to Success: Follow contract requirements and update Execution Plan to account for differences between Basic Design and current conditions. Provide the early resources required to maximize benefits as planned.

- Pitfalls: Blindly follow recommendations without verification of assumptions. Failing to recognize when slipping back to stick-built comfort zone

\section{CONCLUSIONS AND RECOMMENDATIONS}

The researchers have identified that the industry needs additional guidance on how to achieve wider and more effective use of modularization. To provide this guidance, the researchers and the CII Research Team 283 developed a new modularization business case process to develop the optimum degree of modularization for a project. This paper first presented a model business case decision flow chart, followed by a detailed discussion of analysis activities related to modularization by phase. The researchers recommend that consideration of modularization options should be guided by past experience as well as creative identification of options and new approaches given changes in technology, project-specific demands, and so forth. Furthermore, the flowchart detailed in Figure 1 is meant to be repeatedly utilized during each of the major project decision phases, typically in parallel with the development of stick build and modular option estimate data, adding detail in each phase. The findings provide guidelines that should impose rigor on the decision process, help owners, and avoid poor outcomes.

\section{ACKNOWLEDGEMENTS}

This research was sponsored by CII via Research Team 283: Industrial modularization. The authors wish to thank the research team and External Validation Committee for their support.

\section{REFERENCES}

CII (1986). "Control of Construction Project Scope." The University of Texas at Austin: Construction Industry Institute, Austin, TX, 113. SD-6

CII (2012). "Industrial Modularization: How to Optimize; How to Maximize." The University of Texas at Austin: Construction Industry Institute, Austin, TX. RS-283

CII (2013). "Industrial Modularization: Five Solution Elements." The University of Texas at Austin: Construction Industry Institute, Austin, TX. IR-283

Jameson, P. H. (2007). "Is modularization right for your project?" Hydrocarbon Processing, 4753.

O'Connor, J. T., O'Brien, W. J., and Choi, J. O. (2013). "Industrial Modularization: How to Optimize; How to Maximize." The University of Texas at Austin: Construction Industry Institute, Austin, TX. RR-283

Wu, Y., and Lu, M. "Modularization Program Execution Optimization on Mega Oil Sands Projects." Proc., 57th Annual Meeting of the AACE International 2013, AACE International, the Authority for Total Cost Management. 